PROCEEDINGS OF THE

AMERICAN MATHEMATICAL SOCIETY

Volume 139, Number 6, June 2011, Pages 2257-2270

S 0002-9939(2010)10738-4

Article electronically published on November 19, 2010

\title{
ALMOST MAXIMAL TOPOLOGIES ON SEMIGROUPS
}

\author{
YEVHEN ZELENYUK
}

(Communicated by Alexander N. Dranishnikov)

\begin{abstract}
A topology on a semigroup is left invariant if left translations are continuous and open. We show that for every infinite cancellative semigroup $S$ and $n \in \mathbb{N}$, there is a zero-dimensional Hausdorff left invariant topology on $S$ with exactly $n$ nonprincipal ultrafilters converging to the same point, all of them being uniform.
\end{abstract}

\section{INTRODUCTION}

A topological space is called maximal if it has no isolated point but it does have an isolated point in any stronger topology. A Hausdorff space $X$ is maximal if and only if for every point $x \in X$, there is exactly one nonprincipal ultrafilter on $X$ converging to $x$. We say that a space $X$ is almost maximal if it has no isolated point and for every $x \in X$ there are only finitely many nonprincipal ultrafilters on $X$ converging to $x$. A space $X$ is homogeneous if for every $x, y \in X$ there is a homeomorphism $f: X \rightarrow X$ with $f(x)=y$. All topologies are assumed to satisfy the $T_{1}$ separation axiom.

A topology $\mathcal{T}$ on a semigroup $S$ is left invariant if for each $a \in S$, the left translation $S \ni x \mapsto a x \in S$ is continuous and open in $\mathcal{T}$. Equivalently, $\mathcal{T}$ is left invariant if for each $U \in \mathcal{T}$ and $a \in S$, both $a U \in \mathcal{T}$ and $a^{-1} U \in \mathcal{T}$, where

$$
a^{-1} U=\{x \in S: a x \in U\} .
$$

If $S$ has identity 1, a left invariant topology on $S$ is completely determined by the neighborhood filter of 1 . For each $a \in S$, the subsets $a U$, where $U$ runs over a neighborhood base at 1 , form a neighborhood base at $a$. If $S$ is commutative, we say it is translation invariant instead of left invariant.

In this paper we study almost maximal left invariant topologies on semigroups. We show that for every infinite cancellative semigroup $S$ and $n \in \mathbb{N}$, there is a zero-dimensional left invariant topology on $S$ with exactly $n$ nonprincipal ultrafilters on $S$ converging to the same point, all of them being uniform. Recall that a semigroup is cancellative if both left and right translations are injective. A topology is zero-dimensional if it has a base of clopen sets. An ultrafilter $p$ on $S$ is uniform if for every $A \in p,|A|=|S|$. Since a left invariant topology on a group is

Received by the editors January 26, 2010 and, in revised form, June 7, 2010.

2010 Mathematics Subject Classification. Primary 22A05, 54G05; Secondary 22A30, 54H11.

Key words and phrases. Almost maximal space, left invariant topology, Stone-Čech compactification, right maximal idempotent, ultrafilter semigroup, projective.

This work was supported by NRF grant FA2007041200005 and The John Knopfmacher Centre for Applicable Analysis and Number Theory.

(C)2010 American Mathematical Society Reverts to public domain 28 years from publication 2257 
homogeneous, it follows that, for every infinite cardinal $\kappa$ and $n \in \mathbb{N}$, there exists a homogeneous zero-dimensional space of cardinality $\kappa$ with exactly $n$ nonprincipal ultrafilters converging to the same point, all of them being uniform.

The precise statement and the proof of the main result involve the Stone-Cech compactification $\beta S$ of a discrete semigroup $S$. We take the points of $\beta S$ to be the ultrafilters on $S$, the principal ultrafilters being identified with the points of $S$, and we write $S^{*}$ and $U(S)$ for the sets of nonprincipal and uniform ultrafilters on $S$, respectively. The topology of $\beta S$ is generated by taking as a base the subsets

$$
\bar{A}=\{p \in \beta S: A \in p\},
$$

where $A \subseteq S$. For $p, q \in \beta S$, the ultrafilter $p q$ has a base consisting of subsets

$$
\bigcup\left\{x B_{x}: x \in A\right\},
$$

where $A \in p$ and $B_{x} \in q$. Under this operation, $\beta S$ is a compact Hausdorff right topological semigroup with $S$ contained in its topological center. A semigroup $T$ endowed with a topology is right topological if for each $p \in T$, the right translation

$$
T \ni x \mapsto x p \in T
$$

is continuous. The topological center $\Lambda(T)$ of a right topological semigroup $T$ consists of all $a \in T$ such that the left translation

$$
T \ni x \mapsto a x \in T
$$

is continuous. An elementary introduction to the semigroup $\beta S$ can be found in [3].

Now let $S$ be a semigroup with identity 1 and let $\mathcal{T}$ be a left invariant topology on $S$. Define $\operatorname{Ult}(\mathcal{T}) \subseteq S^{*}$ by

$$
\operatorname{Ult}(\mathcal{T})=\bigcap_{U \in \mathcal{N}} \overline{U \backslash\{1\}}
$$

where $\mathcal{N}$ is the neighborhood filter of 1 in $\mathcal{T}$, or equivalently,

$$
\operatorname{Ult}(\mathcal{T})=\left\{p \in S^{*}: p \text { converges to } 1 \text { in } \mathcal{T}\right\} .
$$

Then $\operatorname{Ult}(\mathcal{T})$ is a closed subsemigroup of $\beta S$ called the ultrafilter semigroup of $\mathcal{T}$.

Not every closed subsemigroup in $S^{*}$ is the ultrafilter semigroup of a left invariant topology. However, every finite subsemigroup is.

Lemma 1.1. For every finite semigroup $F$ in $S^{*}$, there is a left invariant topology $\mathcal{T}$ on $S$ such that $\operatorname{Ult}(\mathcal{T})=F$.

Proof. Let $\mathcal{F}$ be the intersection of all ultrafilters from $F$ so that

$$
\bigcap_{A \in \mathcal{F}} \bar{A}=F \text {. }
$$

For every $x \in S$, let $\mathcal{N}_{x}$ denote the filter on $S$ with a base consisting of subsets of the form $x A \cup\{x\}$, where $A \in \mathcal{F}$. We claim that $\left\{\mathcal{N}_{x}: x \in S\right\}$ is the neighborhood system for a left invariant topology $\mathcal{T}$ on $S$. To show this, we have to verify that

(i) for every $x \in S, \cap \mathcal{N}_{x}=\{x\}$,

(ii) for every $x \in S$ and $U \in \mathcal{N}_{x},\left\{y \in S: U \in \mathcal{N}_{y}\right\} \in \mathcal{N}_{x}$, and

(iii) for every $x, y \in S, y \mathcal{N}_{x}$ is a base for $\mathcal{N}_{y x}$. 
Statements (i) and (iii) are obvious. To check (ii), let $x \in S$ and $U \in \mathcal{N}_{x}$. Then $x^{-1} U \in \mathcal{F}$. For every $p, q \in F$, pick $A_{p, q} \in p$ such that $A_{p, q} \subseteq x^{-1} U$ and $\overline{A_{p, q}} \cdot q \subseteq \overline{x^{-1} U}$. Put $A=\bigcup_{p \in F} \bigcap_{q \in F} A_{p, q}$. Then $A \in \mathcal{F}, A \subseteq x^{-1} U$ and $A F \subseteq \overline{x^{-1} U}$, so $x A \subseteq U$ and $x A F \subseteq \bar{U}$. Define $V \in \mathcal{N}_{x}$ by $V=x A \cup\{x\}$. We claim that for every $y \in V$, there is $W_{y} \in \mathcal{N}_{y}$ such that $W_{y} \subseteq U$.

Indeed, if $y=x$, put $W_{y}=V$. Otherwise $y \in x A$. For every $q \in F$, pick $B_{y, q} \in q$ such that $y B_{y, q} \subseteq U$. Put $B_{y}=\bigcup_{q \in F} B_{y, q}$. Then $B_{y} \in \mathcal{F}$ and $y B_{y} \subseteq U$. Define $W_{y} \in \mathcal{N}_{y}$ by $W_{y}=y B_{y} \cup\{y\}$.

Thus, there is a one-to-one correspondence between almost maximal left invariant topologies on $S$ and finite semigroups in $S^{*}$. Among the latter, the most common are bands, that is, semigroups of idempotents. The simplest examples of bands are left zero semigroups, defined by the identity $x y=x$, right zero semigroups, defined by the identity $x y=y$, and chains of idempotents, with respect to the order $x \leq y$ if and only if $x y=y x=x$. The direct product of a left zero semigroup and a right zero semigroup is called a rectangular semigroup. Each band is a disjoint union of its maximal rectangular subsemigroups called rectangular components, and these are partially ordered by the relation $P \leq Q$ if and only if $P Q \subseteq P$, equivalently $Q P \subseteq P$ [5, Theorem 1].

The aim of this paper is to show that

Theorem 1.2. For every infinite cancellative semigroup $S$ with identity and for every $n \in \mathbb{N}$, there is a zero-dimensional left invariant topology $\mathcal{T}$ on $S$ with $\operatorname{Ult}(\mathcal{T})$ being a chain of $n$ idempotents in $U(S)$.

Note that 'with identity' in Theorem 1.2 is not a restriction. If $S$ is cancellative, so is $S^{1}$ [1, Section 1.1, Exercise 2].

Theorem 1.2 has been previously proved in the following cases:

(a) $S$ is a countably infinite group and $n=1$ [7,

(b) $S$ is a countably infinite group [11, and

(c) $n=1$ [14.

The proof of Theorem 1.2 occupies the rest of the paper. Basically, it consists of proving that there is a locally zero-dimensional translation invariant topology on the Boolean group with the ultrafilter semigroup being a chain of $n$ uniform idempotents. A space is locally zero-dimensional if every point has a neighborhood which is a zero-dimensional subspace. Theorem 1.2 is a consequence of this result and the so-called Local Monomorphism Theorem [14, Theorem 6.4].

\section{The ultrafilter Semigroup \\ OF AN ALMOST MAXIMAL LEFT INVARIANT TOPOLOGY}

Throughout the rest of the paper, we will use the following notation.

Definition 2.1. Let $\kappa$ be an infinite cardinal and let $G=\bigoplus_{\kappa} \mathbb{Z}_{2}$. For each $\alpha<\kappa$, let $G_{\alpha}=\{x \in G: x(\gamma)=0$ for all $\gamma<\alpha\}$, and let $\mathcal{G}$ denote the group topology on $G$ with a neighborhood base at 0 consisting of subgroups $G_{\alpha}$, where $\alpha<\kappa$. The semigroup $\mathbb{H}_{\kappa}$ is defined by $\mathbb{H}_{\kappa}=\operatorname{Ult}(\mathcal{G})$.

The semigroup $\mathbb{H}_{\kappa}$ enjoys remarkable properties. In particular, every compact right topological semigroup $T$ containing a dense subset $A$ such that $|A| \leq \kappa$ and $A \subseteq \Lambda(T)$ is a continuous homomorphic image of $\mathbb{H}_{\kappa}$, 4, Theorem 2.5], and for 
every cancellative semigroup $S$ of cardinality $\kappa$, there are copies of $\mathbb{H}_{\kappa}$ in $S^{*}$, Theorem 2.7]. In fact, the second result can be a little bit strengthened.

Theorem 2.2. Let $S$ be an infinite cancellative semigroup with identity and let $|S|=\kappa$. Then there is a zero-dimensional left invariant topology $\mathcal{T}$ on $S$ such that $\operatorname{Ult}(\mathcal{T}) \subseteq U(S)$ and $\operatorname{Ult}(\mathcal{T})$ is topologically and algebraically isomorphic to $\mathbb{H}_{\kappa}$.

The proof of Theorem 2.2 is based on the following lemma.

Lemma 2.3. Let $S$ be an infinite cancellative semigroup with identity and let $|S|=$ $\kappa$. Then there are two $\kappa$-sequences $\left(x_{\alpha}\right)_{1 \leq \alpha<\kappa}$ and $\left(y_{\alpha}\right)_{\alpha<\kappa}$ in $S$ with $y_{0}=1$ such that every element of $S$ is uniquely representable in the form $y_{\alpha_{0}} x_{\alpha_{1}} \cdots x_{\alpha_{n}}$, where $n<\omega$ and $\alpha_{0}<\alpha_{1}<\ldots<\alpha_{n}<\kappa$.

Proof. Enumerate $S$ as $\left\{s_{\alpha}: \alpha<\kappa\right\}$. Put $y_{0}=1$. Fix $0<\gamma<\kappa$ and suppose that we have constructed $\left(x_{\alpha}\right)_{1 \leq \alpha<\gamma}$ and $\left(y_{\alpha}\right)_{\alpha<\gamma}$ such that all products $y_{\alpha_{0}} x_{\alpha_{1}} \cdots x_{\alpha_{n}}$, where $n<\omega$ and $\alpha_{0}<\alpha_{1}<\ldots<\alpha_{n}<\gamma$, are different. Pick as $y_{\gamma}$ the first element in the sequence $\left(s_{\alpha}\right)_{\alpha<\kappa}$ not belonging to the subset

$$
S_{\gamma}=\left\{y_{\alpha_{0}} x_{\alpha_{1}} \cdots x_{\alpha_{n}}: n<\omega \text { and } \alpha_{0}<\alpha_{1}<\ldots<\alpha_{n}<\gamma\right\} .
$$

Then pick $x_{\gamma} \in S \backslash\left(S_{\gamma}^{-1} S_{\gamma}\right)$. (Here, $S_{\gamma}^{-1} S_{\gamma}=\bigcup_{x \in S_{\gamma}} x^{-1} S_{\gamma}$.) This can be done because $\left|S_{\gamma}^{-1} S_{\gamma}\right| \leq\left|S_{\gamma}\right|^{2}<\kappa$. Then whenever $n<\omega$ and $\alpha_{0}<\alpha_{1}<\ldots<\alpha_{n}=\gamma$, one has $y_{\alpha_{0}} x_{\alpha_{1}} \cdots x_{\alpha_{n}} \notin S_{\gamma}$. Also if $y_{\alpha_{0}} x_{\alpha_{1}} \cdots x_{\alpha_{n}}$ and $y_{\beta_{0}} x_{\beta_{1}} \cdots x_{\beta_{m}}$ are different elements of $S_{\gamma}$, the elements $y_{\alpha_{0}} x_{\alpha_{1}} \cdots x_{\alpha_{n}} x_{\gamma}$ and $y_{\beta_{0}} x_{\beta_{1}} \cdots x_{\beta_{m}} x_{\gamma}$ are different as well.

Proof of Theorem 2.2. Let $\left(x_{\alpha}\right)_{1 \leq \alpha<\kappa}$ and $\left(y_{\alpha}\right)_{\alpha<\kappa}$ be sequences guaranteed by Lemma 2.3. Define $\varphi: S \rightarrow \kappa$ by

$$
\varphi\left(y_{\alpha_{0}} x_{\alpha_{1}} \cdots x_{\alpha_{n}}\right)=\alpha_{n},
$$

where $n<\kappa$ and $\alpha_{0}<\alpha_{1}<\ldots<\alpha_{n}<\kappa$. Then for every $x \in S$ and $\varphi(x)<\alpha<\kappa$, define $B(x, \alpha) \subseteq S$ by

$$
B(x, \alpha)=\left\{x x_{\alpha_{1}} \cdots x_{\alpha_{n}}: n<\omega, \alpha \leq \alpha_{1}<\ldots<\alpha_{n}<\kappa\right\} .
$$

The subsets $B(x, \alpha)$ possess the following properties:

(i) for every $x \in S,(B(x, \alpha))_{\varphi(x)<\alpha<\kappa}$ is a decreasing sequence of subsets of $S$ with $\bigcap_{\varphi(x)<\alpha<\kappa} B(x, \alpha)=\{x\}$

(ii) whenever $x, y \in S, x \neq y, \varphi(x)<\alpha<\kappa$ and $\varphi(y)<\gamma<\kappa$, one has $B(y, \gamma) \subseteq B(x, \alpha)$ if $y \in B(x, \alpha)$ and $B(y, \gamma) \cap B(x, \alpha)=\emptyset$ otherwise;

(iii) whenever $x, y \in S$ and $\max \{\varphi(y), \varphi(x y)\}<\gamma<\kappa$, one has $x B(y, \gamma)=$ $B(x y, \gamma)$.

It follows that $\{B(x, \alpha): x \in S, \varphi(x)<\alpha<\kappa\}$ is a base for a zero-dimensional left invariant topology $\mathcal{T}$ on $S$.

Clearly $\operatorname{Ult}(\mathcal{T}) \subseteq U(S)$. To see that $\operatorname{Ult}(\mathcal{T})$ is topologically and algebraically isomorphic to $\mathbb{H}_{\kappa}$, let $B=B(1,1)$. Define $f: B \rightarrow G$ by

$$
\operatorname{supp}\left(f\left(y_{0} x_{\alpha_{1}} \cdots x_{\alpha_{n}}\right)\right)=\left\{\alpha_{1}, \ldots, \alpha_{n}\right\}
$$

where $n<\omega$ and $0<\alpha_{1}<\ldots<\alpha_{n}<\kappa$. As usual, for every $z \in G$,

$$
\operatorname{supp}(z)=\{\alpha<\kappa: z(\alpha) \neq 0\}
$$

Let $\bar{f}: c \ell_{\beta S} B \rightarrow \beta G$ denote the continuous extension of $f$. Then $\left.\bar{f}\right|_{\operatorname{Ult}(\mathcal{T})}$ : $\operatorname{Ult}(\mathcal{T}) \rightarrow \mathbb{H}_{\kappa}$ is a topological and algebraic isomorphism. 
Remark 2.4. It is easy to see that the condition 'cancellative' in Lemma 2.3 and Theorem 2.2 may be replaced by the following weaker one: every system of $<\kappa$ inequalities over $S$ of the form $a x \neq b$, where $a, b \in S$, or $a x \neq b x$, where $a, b \in S$ and $a \neq b$, has a solution in $S$.

Now we shall show how one can construct Hausdorff almost maximal left invariant topologies.

We say that an object $P$ in some category is an absolute coretract if for every surjective morphism $g: R \rightarrow P$ there exists a morphism $h: P \rightarrow R$ such that $g \circ h=\operatorname{id}_{P}$. Let $\mathfrak{C}$ denote the category of compact Hausdorff right topological semigroups.

Theorem 2.5. Let $S$ be an infinite cancellative semigroup with identity, let $P$ be a finite absolute coretract in $\mathfrak{C}$, and let $F$ be a subsemigroup of $P$. Then there is a left invariant Hausdorff topology $\mathcal{T}$ on $S$ such that $\operatorname{Ult}(\mathcal{T}) \subseteq U(S)$ and $\operatorname{Ult}(\mathcal{T})$ is isomorphic to $F$.

Proof. By Theorem 2.2, there is a zero-dimensional left invariant topology $\mathcal{T}_{0}$ on $S$ such that $\operatorname{Ult}\left(\mathcal{T}_{0}\right) \subseteq U(S)$ and $\operatorname{Ult}\left(\mathcal{T}_{0}\right)$ is topologically and algebraically isomorphic to $\mathbb{H}_{\kappa}$. Let $R=\operatorname{Ult}\left(\mathcal{T}_{0}\right)$. Then there is a surjective continuous homomorphism $g: R \rightarrow P$. Consequently, there is an injective homomorphism $h: P \rightarrow R$ (such that $\left.g \circ h=\mathrm{id}_{P}\right)$. Let $Q=h(F)$. Then there is a left invariant topology $\mathcal{T}$ on $S$ such that $\operatorname{Ult}(\mathcal{T})=Q$. We have that $\operatorname{Ult}(\mathcal{T}) \subseteq U(S)$, $\operatorname{Ult}(\mathcal{T})$ is isomorphic to $F$ and $\mathcal{T}$ is Hausdorff, since $\mathcal{T}_{0} \subseteq \mathcal{T}$.

To construct regular almost maximal left invariant topologies is much harder than Hausdorff ones. First, we shall study their ultrafilter semigroups. This, in turn, involves the notion of a local homomorphism.

Let $S$ be a semigroup with identity, let $\mathcal{T}$ be a left invariant topology on $S$, and let $X$ be an open neighborhood of 1 in $\mathcal{T}$. A mapping $f: X \rightarrow R$ of $X$ into a semigroup $R$ is a local homomorphism if for every $x \in X \backslash\{1\}$, there is a neighborhood $U$ of $1 \in X$ such that $f(x y)=f(x) f(y)$ for all $y \in U \backslash\{1\}$. If $R$ has identity, we require in addition that $f\left(1_{S}\right)=1_{R}$. An injective (bijective) local homomorphism is called a local monomorphism (a local isomorphism).

Local homomorphisms are important because of the following fact:

If $f: X \rightarrow T$ is a local homomorphism into a compact right topological semigroup $T$ such that $f(X) \subseteq \Lambda(T), \bar{f}: c \ell_{\beta S} X \rightarrow T$ is the continuous extension of $f$ and $f^{*}=\left.\bar{f}\right|_{\operatorname{Ult}(\mathcal{T})}$, then $f^{*}: \operatorname{Ult}(\mathcal{T}) \rightarrow T$ is a homomorphism [14, Lemma 2.12].

A remarkable property of local homomorphisms is contained in the next proposition.

Proposition 2.6 ([12, Proposition 3.4]). Let $\mathcal{T}$ be any zero-dimensional left invariant topology on $G$ such that $\mathcal{G} \subseteq \mathcal{T}$ and let $X$ be an open neighborhood of zero in $\mathcal{T}$. Then for every homomorphism $g: R \rightarrow Q$ of a semigroup $R$ onto a semigroup $Q$ and for every local homomorphism $f: X \rightarrow Q$, there is a local homomorphism $h: X \rightarrow R$ such that $f=g \circ h$.

Recall that an object $P$ in some category is a projective if for every morphism $f: P \rightarrow Q$ and for every surjective morphism $g: R \rightarrow Q$, there exists a morphism $h: P \rightarrow R$ such that $g \circ h=f$. Obviously, each projective is an absolute coretract. In many categories these notions coincide, but not in all. Let $\mathfrak{F}$ denote the category of finite semigroups. 
We use Proposition 2.6 to prove the following result.

Theorem 2.7. Let $\mathcal{T}$ be a zero-dimensional almost maximal left invariant topology on $G$ such that $\mathcal{G} \subseteq \mathcal{T}$. Then $\operatorname{Ult}(\mathcal{T})$ is a projective in $\mathfrak{F}$.

Proof. Let $P=\operatorname{Ult}(\mathcal{T})$, let $R$ and $Q$ be finite semigroups, let $f: P \rightarrow Q$ and $g: R \rightarrow Q$ be homomorphisms, and let $g$ be surjective.

For each $p \in S$, choose $A_{p} \in p$ such that $A_{p} \cap A_{q}=\emptyset$ if $p \neq q$. Then, for each $p \in S$, choose $B_{p} \in p$ such that $\overline{B_{p}}+q \subseteq \overline{A_{p+q}}$ for all $q \in S$. This can be done, since the mapping $\beta G \ni x \mapsto x+q \in \beta G$ is continuous and $S$ is finite. Choose the subsets $B_{p}$ in addition so that $B_{p} \subseteq A_{p}$ and $X=\bigcup_{p \in S} B_{p} \cup\{0\}$ is open in $\mathcal{T}$. Define $f_{0}: X \rightarrow Q$ putting for every $p \in S$ and $x \in B_{p}, f_{0}(x)=f(p)$. The value $f_{0}(0)$ does not matter. We claim that $f_{0}$ is a local homomorphism and $f_{0}^{*}=f$.

It suffices to check the first statement. Let $x \in X \backslash\{0\}$. Then $x \in B_{p}$ for some $p \in S$. For each $q \in S$, choose $D_{q} \in q$ such that $D_{q} \subseteq A_{q}$ and $x+D_{q} \subseteq A_{p+q}$. Choose a neighborhood $U$ of $0 \in X$ such that $U \subseteq \bigcup_{q \in S} D_{q} \cup\{0\}$ and $x+U \subseteq X$. Now let $y \in U \backslash\{0\}$. Then $y \in D_{q}$ for some $q \in S$, so $y \in A_{q}$ and then $y \in B_{q}$. Hence $f_{0}(x) f_{0}(y)=f(p) f(q)$. On the other hand, $x+y \in A_{p+q}$, then $x+y \in B_{p+q}$, and so

$$
f_{0}(x+y)=f(p+q)=f(p) f(q) .
$$

Hence $f_{0}(x+y)=f_{0}(x) f_{0}(y)$.

By Proposition 2.6. there is a local homomorphism $h_{0}: X \rightarrow R$ such that $f_{0}=g \circ h_{0}$. Put $h=h_{0}^{*}$. Since

$$
\left(g \circ h_{0}\right)^{*}=\left.\overline{g \circ h_{0}}\right|_{S}=\left.g \circ \overline{h_{0}}\right|_{S}=g \circ h,
$$

we obtain that

$$
f=f_{0}^{*}=\left(g \circ h_{0}\right)^{*}=g \circ h .
$$

We conclude this section by characterizing finite absolute coretracts in $\mathfrak{C}$ and projectives in $\mathfrak{F}$, following [9] and [10].

Let $V$ denote the set of words of the form

$$
i_{1} i_{2} \ldots i_{p} \lambda_{p} \lambda_{p-1} \ldots \lambda_{1}
$$

where $p \in \mathbb{N}$ and $i_{q}, \lambda_{q} \in \omega$ for all $q=1, \ldots, p$. Define the operation on $V$ as follows:

$$
i_{1} \ldots i_{p} \lambda_{p} \ldots \lambda_{1} \cdot j_{1} \ldots j_{q} \rho_{q} \ldots \rho_{1}= \begin{cases}i_{1} \ldots i_{p} \rho_{p} \ldots \rho_{1} & \text { if } p=q \\ i_{1} \ldots i_{p} \lambda_{p} \ldots \lambda_{q+1} \rho_{q} \ldots \rho_{1} & \text { if } p>q \\ i_{1} \ldots i_{p} j_{p+1} \ldots j_{q} \rho_{q} \ldots \rho_{1} & \text { if } p<q .\end{cases}
$$

Then $V$ is a band being decomposed into a decreasing chain of its rectangular components $V_{p}$ whose elements are words of length $2 p$. For every subsemigroup $W$ of $V$, put $W_{p}=W \cap V_{p}$.

If $v=i_{1} \ldots i_{p} \lambda_{p} \ldots \lambda_{1} \in V_{p}$, let $v^{\prime}=i_{1} \ldots i_{p}$ and $v^{\prime \prime}=\lambda_{p} \ldots \lambda_{1}$, and for every $q=1, \ldots, p$, let $v_{q}^{\prime}=i_{q}$ and $v_{q}^{\prime \prime}=\lambda_{q}$.

Now let $\mathcal{P}$ denote the class of finite subsemigroups $W$ of $V$ such that for every $p \in \mathbb{N}$, the following conditions are satisfied:

(i) if $v \in W_{p}$, both $v_{p}^{\prime} \neq 0$ and $v_{p}^{\prime \prime} \neq 0$; 
(ii) if $v \in W_{p}$ and $v_{q}^{\prime} \neq 0$ for some $q<p$, there exists $w \in W_{q}$ such that $w^{\prime}$ is the initial segment of $v^{\prime}$, and dually, if $v \in W_{p}$ and $v_{q}^{\prime \prime} \neq 0$ for some $q<p$, there exists $w \in W_{q}$ such that $w^{\prime \prime}$ is the final segment of $v^{\prime \prime}$;

(iii) either $v_{p}^{\prime}=1$ for all $v \in W_{q}$ with $q \geq p$ or $v_{q}^{\prime \prime}=1$ for all $v \in W_{q}$ with $q \geq p$.

To give a simple important example, let $\left(m_{p}\right)_{p=1}^{l}$ and $\left(n_{p}\right)_{p=1}^{l}$ be two sequences in $\mathbb{N}$ of the same finite length $l$ such that for each $p$, either $m_{p}=1$ or $n_{p}=1$. Denote by $W\left[\left(m_{p}\right)_{p=1}^{l},\left(n_{p}\right)_{p=1}^{l}\right]$ the subset of $V$ consisting of all words of the form

$$
i_{1} i_{2} \ldots i_{p} \lambda_{p} \lambda_{p-1} \ldots \lambda_{1}
$$

where $p=1, \ldots, l$, and for each $q=1, \ldots, p, i_{q} \in\left\{1, \ldots, m_{p}\right\}$ and $\lambda_{q} \in\left\{1, \ldots, n_{p}\right\}$. It is easy to see that $W\left[\left(m_{p}\right)_{p=1}^{l},\left(n_{p}\right)_{p=1}^{l}\right]$ is a semigroup from $\mathcal{P}$. Note that every finite subsemigroup of $V$ can be isomorphically embedded into a semigroup $W\left[\left(m_{p}\right)_{p=1}^{l},\left(n_{p}\right)_{p=1}^{l}\right]$.

Theorem 2.8 ([10]). Let $F$ be a finite semigroup. Then the following statements are equivalent:

(1) F belongs to $\mathcal{P}$.

(2) $F$ is a projective in $\mathfrak{F}$.

(3) $F$ is an absolute coretract in $\mathfrak{F}$.

(4) $F$ is a projective in $\mathfrak{C}$.

(5) $F$ is an absolute coretract in $\mathfrak{C}$.

Recall that Green's relations $\mathcal{R}$ and $\mathcal{L}$ on a semigroup $S$ are defined by

$$
a \mathcal{R} b \Leftrightarrow a S^{1}=b S^{1} \text { and } a \mathcal{L} b \Leftrightarrow S^{1} a=S^{1} b .
$$

Note that elements of a band are $\mathcal{R}$-related $(\mathcal{L}$-related) if and only if they belong to the same rectangular component and to the same minimal right (left) ideal of the component.

We shall need the following consequence of Theorem 2.8 .

Proposition 2.9. Every projective $P$ in $\mathfrak{F}$ is a chain of rectangular bands satisfying the following conditions:

(i) whenever $x, y, z \in P$ and $y \mathcal{R} z, x y=x z$ implies $y=z$; and dually

(ii) whenever $x, y, z \in P$ and $y \mathcal{L} z, y x=z x$ implies $y=z$.

\section{The Semigroup $C(p)$}

Definition 3.1. Given a semigroup $S$ and $p \in S^{*}$,

$$
C(p)=\left\{x \in S^{*}: x p=p\right\} .
$$

Note that if $S^{*}$ is a subsemigroup of $\beta S$, then $C(p)$ is a closed subsemigroup of $S^{*}$ if it is nonempty, and $p \in C(p)$ if and only if $p$ is an idempotent. If $S$ has identity, we use $C^{1}(p)$ to denote $C(p) \cup\{1\} \subseteq \beta S$. If $S$ has identity and is cancellative, $C^{1}(p)=\{x \in \beta S: x p=p\}$ [14, Lemma 2.9]. Also note that for every $p \in \mathbb{H}_{\kappa}$, $C(p) \subseteq \mathbb{H}_{\kappa}$.

The next lemma explains why this semigroup is important.

Lemma $3.2([6])$. Let $S$ be a group and let $p \in S^{*}$. Then there is a zero-dimensional left invariant topology $\mathcal{T}$ on $S$ with $\operatorname{Ult}(\mathcal{T})=C(p)$. 
In [14, it was shown that there are idempotents $p \in \mathbb{H}_{\kappa}$ with finite $C(p)$. Consequently, by Lemma 3.2. there are zero-dimensional almost maximal translation invariant topologies $\mathcal{T}$ on $G$ with $\operatorname{Ult}(\mathcal{T}) \subseteq \mathbb{H}_{\kappa}$. To state the result from [14] precisely, we need several more definitions.

There are standard right and left preorderings and order on idempotents of any semigroup. These are defined by

$$
\begin{aligned}
x \leq_{R} y & \Leftrightarrow x=y x, \\
x \leq_{L} y & \Leftrightarrow x=x y, \text { and } \\
x \leq y & \Leftrightarrow x=y x=x y .
\end{aligned}
$$

An idempotent $p$ of a semigroup $S$ is right maximal if for every idempotent $q \in S$, $p \leq_{R} q$ implies $q \leq_{R} p$. Every compact Hausdorff right topological semigroup has a right maximal idempotent [8, Theorem 2.7].

An ultrafilter $u$ on $\kappa$ is countably complete if whenever $\left\{A_{n}: n<\omega\right\}$ is a partition of $\kappa$, there is $n<\omega$ such that $A_{n} \in u$. A cardinal $\kappa$ is Ulam-measurable if there is a countably complete nonprincipal ultrafilter on $\kappa$. It is consistent with ZFC that there is no Ulam-measurable cardinal. (See [2, Section 8].)

Define the functions $\theta, \phi: G \backslash\{0\} \rightarrow \kappa$ by

$$
\theta(x)=\min \operatorname{supp}(x) \text { and } \phi(x)=\max \operatorname{supp}(x)
$$

and let $\bar{\theta}, \bar{\phi}: \beta G \backslash\{0\} \rightarrow \beta \kappa$ be their continuous extensions. The main properties of these functions are that for every $x \in \beta G \backslash\{0\}$ and $y \in \mathbb{H}_{\kappa}$,

$$
\bar{\theta}(x+y)=\bar{\theta}(x) \text { and } \bar{\phi}(x+y)=\bar{\phi}(y) \text {. }
$$

Theorem 3.3 ([14, Theorem 5.1]). Let $p$ be a right maximal idempotent in $\mathbb{H}_{\kappa}$. Then $C(p)$ is a compact right zero semigroup, and if the ultrafilter $\bar{\theta}(p)$ on $\kappa$ is countably incomplete, $C(p)$ is finite.

It follows from Theorem 3.3 that, whenever $\kappa$ is an infinite cardinal, there is a right maximal idempotent $p \in \mathbb{H}_{\kappa}$ such that $C(p)$ is a finite right zero semigroup, and if $\kappa$ is not Ulam-measurable, every right maximal idempotent $p \in \mathbb{H}_{\kappa}$ enjoys this property.

Now we shall prove the following result.

Theorem 3.4. Let $p \in \mathbb{H}_{\kappa}$ and let $C(p)$ be finite. Then

(1) $C(p)$ is a projective in $\mathfrak{F}$, and

(2) $C(p)$ is a chain of right zero semigroups.

(1) is immediate from Lemma 3.2 and Theorem 2.2, To prove (2), we need the following lemma.

Lemma 3.5. Let $p \in \mathbb{H}_{\kappa}$ and let $C(p)$ be finite. Then for every $q, r \in \beta G$, the equality $q+p=r+p$ implies that $q \in r+C^{1}$ or $r \in q+C^{1}$, where $C^{1}=C^{1}(p)$.

Proof. Assume the contrary. Then, since $C^{1}$ is finite, there exist $A \in q$ and $B \in r$ such that

$$
\bar{A} \cap\left(B+C^{1}\right)=\emptyset \text { and } \bar{B} \cap\left(A+C^{1}\right)=\emptyset .
$$

By Lemma 3.2 there is a left invariant topology $\mathcal{T}$ on $G$ with $\operatorname{Ult}(\mathcal{T})=C(p)$. It follows that for every $x \in A \cup B$, there exists a neighborhood $U$ of $0 \in G$ in $\mathcal{T}$ such that

$$
A \cap(x+U)=\emptyset \text { if } x \in B \text {, and } B \cap(x+U)=\emptyset \text { if } x \in A \text {. }
$$


Since $\mathcal{T}$ is regular, the neighborhoods can be chosen to be closed.

Enumerate $A \cup B$ as $\left\{x_{\alpha}: \alpha<\kappa\right\}$ so that the sequence $\left(\phi\left(x_{\alpha}\right)\right)_{\alpha<\kappa}$ is nondecreasing. For each $\alpha<\kappa$, choose inductively a closed neighborhood $U_{\alpha}$ of 0 in $\mathcal{T}$ so that the following conditions are satisfied:

(i) $U_{\alpha} \subseteq G_{\phi\left(x_{\alpha}\right)+1}$,

(ii) $A \cap\left(x_{\alpha}+U_{\alpha}\right)=\emptyset$ if $x_{\alpha} \in B$, and $B \cap\left(x_{\alpha}+U_{\alpha}\right)=\emptyset$ if $x_{\alpha} \in A$, and

(iii) $\left(x_{\alpha}+U_{\alpha}\right) \cap\left(x_{\gamma}+U_{\gamma}\right)=\emptyset$ for all $\gamma<\alpha$ such that $\operatorname{supp}\left(x_{\gamma}\right) \subseteq \operatorname{supp}\left(x_{\alpha}\right)$ and elements $x_{\alpha}, x_{\gamma}$ belong to different sets $A, B$.

To see that this can be done, fix $\alpha<\kappa$ and suppose that we have already chosen $U_{\gamma}$ for all $\gamma<\alpha$ satisfying (i)-(iii). Without loss of generality one may also suppose that $x_{\alpha} \in A$. Let

$$
F=\left\{\gamma<\alpha: \operatorname{supp}\left(x_{\gamma}\right) \subseteq \operatorname{supp}\left(x_{\alpha}\right) \text { and } x_{\gamma} \in B\right\} .
$$

It follows from (ii) that

$$
x_{\alpha} \notin \bigcup_{\gamma \in F}\left(x_{\gamma}+U_{\gamma}\right) .
$$

Since $F$ is finite and each $U_{\gamma}$ is closed, there is a neighborhood $U_{\alpha}$ of 0 (closed) such that

$$
\left(x_{\alpha}+U_{\alpha}\right) \cap\left(\bigcup_{\gamma \in F}\left(x_{\gamma}+U_{\gamma}\right)\right)=\emptyset,
$$

which means that (iii) is satisfied. Obviously, one can choose $U_{\alpha}$ to also satisfy (i) and (ii).

We now claim that $\left(x_{\alpha}+U_{\alpha}\right) \cap\left(x_{\gamma}+U_{\gamma}\right)=\emptyset$ whenever $\gamma<\alpha<\kappa$ and elements $x_{\alpha}, x_{\gamma}$ belong to different sets $A, B$. Indeed, if $\operatorname{supp}\left(x_{\gamma}\right) \subseteq \operatorname{supp}\left(x_{\alpha}\right)$, then $\left(x_{\alpha}+U_{\alpha}\right) \cap\left(x_{\gamma}+U_{\gamma}\right)=\emptyset$ by (iii). If $\operatorname{supp}\left(x_{\gamma}\right) \backslash \operatorname{supp}\left(x_{\alpha}\right) \neq \emptyset$, then $\left(x_{\alpha}+G_{\phi\left(x_{\alpha}\right)+1}\right) \cap$ $\left(x_{\gamma}+G_{\phi\left(x_{\gamma}\right)+1}\right)=\emptyset$, and consequently, $\left(x_{\alpha}+U_{\alpha}\right) \cap\left(x_{\gamma}+U_{\gamma}\right)=\emptyset$ by (i).

Thus, we have that

$$
\left(\bigcup_{x_{\alpha} \in A}\left(x_{\alpha}+U_{\alpha}\right)\right) \cap\left(\bigcup_{x_{\gamma} \in B}\left(x_{\gamma}+U_{\gamma}\right)\right)=\emptyset
$$

so $q+p \neq r+p$, which is a contradiction.

Given a semigroup $C$, let $K(C)$ denote the smallest ideal of $C$, provided it exists. Note that in the case where $C$ is a finite chain of rectangular bands, say that $C_{1}>\ldots>C_{n}, K(S)=C_{n}$ is the lowest component.

Proof of Theorem 3.4(2). Let $C=C(p)$. By (1) and Proposition 2.9, $C$ is a chain of rectangular bands. We have to show that for every $x, y \in C, x \mathcal{L} y$ implies $x=y$. Let $K=K(C)$. Pick any $z \in K$. Then $x+z, y+z \in K$ and $(x+z) \mathcal{L}(y+z)$. We also have that $x+z+p=y+z+p$. It follows from this and Lemma 3.5 that either $x+z \in y+z+C^{1}$ or $y+z \in x+z+C^{1}$, where $C^{1}=C^{1}(p)$. Both $y+z+C^{1}$ and $x+z+C^{1}$ are $\mathcal{R}$-classes of $K$. Therefore in any case, $(x+z) \mathcal{R}(y+z)$. Since also $(x+z) \mathcal{L}(y+z)$, we obtain that $x+z=y+z$ and then, by Proposition 2.9 (ii), $x=y$.

In the rest of this section, we shall show that for every $n \in \mathbb{N}$, there is an idempotent $p \in \mathbb{H}_{\kappa}$ such that $C(p)$ is a chain of $n$ finite right zero semigroups.

We start with the following consequence of Lemma 3.5 
Lemma 3.6. Let $p \in \mathbb{H}_{\kappa}$ and let $C(p)$ be finite. Then for every $q \in G^{*}$,

$$
|\{x \in \beta G: x+p=q\}| \leq\left|C^{1}(p)\right| .
$$

Proof. Let $X=\{x \in \beta G: x+p=q\}$ and let $C^{1}=C^{1}(p)$. Choose $y \in X$ with maximally possible $\left|y+C^{1}\right|$. For every $z \in C^{1}$, one has $y+z+p=y+p=q$, so $y+C^{1} \subseteq X$. We claim that $X=y+C^{1}$.

To see this, let $x \in X$. We have that $x+p=y+p$. Then by Lemma 3.5, either $x \in y+C^{1}$ or $y \in x+C^{1}$. The first possibility is what we wish to show. The second implies that $y+C^{1} \subseteq x+C^{1}$. Since $\left|y+C^{1}\right|$ is maximally possible, we obtain that $y+C^{1}=x+C^{1}$, so again $x \in y+C^{1}$.

It follows from $X=y+C^{1}$ that $|X| \leq\left|C^{1}\right|$.

Lemma 3.7. Let $p, q \in \mathbb{H}_{\kappa}$ and let $C(p), C(q)$ be finite. Then

$$
\left|C^{1}(p+q)\right| \leq\left|C^{1}(p)\right| \cdot\left|C^{1}(q)\right| .
$$

Proof. We have that

$$
\begin{aligned}
C^{1}(p+q) & =\{x \in \beta G: x+p+q=p+q\} \\
& =\{x \in \beta G: x+p \in\{y \in \beta G: y+q=p+q\}\} .
\end{aligned}
$$

Let $Y=\{y \in \beta G: y+q=p+q\}$, and for each $y \in Y$, let $X(y)=\{x \in \beta G$ : $x+p=y\}$. Then

$$
C^{1}(p+q)=\bigcup_{y \in Y} X(y)
$$

and, by Lemma 3.6. $|Y| \leq\left|C^{1}(q)\right|$ and $|X(y)| \leq C^{1}(p)$.

Now we shall prove the following.

Proposition 3.8. For every idempotent $p \in \mathbb{H}_{\kappa}$ with finite $C(p)$, there is a right maximal idempotent $q \in \mathbb{H}_{\kappa}$ with finite $C(q)$ such that for each $x \in C(q), x<_{L} p$.

The proof of Proposition 3.8 involves some additional notions and results.

Let $S$ be a group and let $p \in S^{*}$. Then there is a largest left invariant topology $\mathcal{T}[p]$ on $S$ in which $p$ converges to 1 . The open neighborhoods of an element $a \in S$ in $\mathcal{T}[p]$ are precisely the subsets of the form

$$
[M]_{a}=\left\{x_{0} \cdots x_{n}: n<\omega, x_{0}=a \text { and } x_{i+1} \in M\left(x_{0} \cdots x_{i}\right) \text { for all } i<n\right\},
$$

where $M: G \rightarrow p$ [13, Proposition 2.2].

Lemma 3.9. Let $p \in S^{*}$ and let $Q=\operatorname{Ult}(\mathcal{T}[p])$. Then $Q=(Q p) \cup\{p\}$.

Proof. Clearly $(Q p) \cup\{p\} \subseteq Q$. We have to show that for every $q \in S^{*} \backslash((Q p) \cup\{p\})$, one has $q \notin Q$. Pick $A \in q$ such that $1 \notin A$ and $\bar{A} \cap((Q p) \cup\{p\})=\emptyset$. It suffices to construct a neighborhood $U$ of 1 in $\mathcal{T}[p]$ such that $U \cap A=\emptyset$.

Since $\bar{A} \cap((Q p) \cup\{p\})=\emptyset$, there is an open neighborhood $V$ of 1 in $\mathcal{T}[p]$ such that $A \cap(\bar{V} p)=\emptyset$. For every $x \in V$, pick $M(x) \in p$ such that $x M(x) \subseteq V$ and $(x M(x)) \cap A=\emptyset$. Put $U=[M]_{1}$. Then $U \subseteq\{1\} \cup \bigcup_{x \in V}(x M(x))$. It follows that $U \cap A=\emptyset$.

Lemma 3.10. For every $p \in \mathbb{H}_{\kappa}$ and $q \in \operatorname{Ult}(\mathcal{T}[p])$, one has $\bar{\theta}(q)=\bar{\theta}(p)$.

Proof. Let $A \in p$. Choose $M: G \rightarrow p$ such that $M(0) \subseteq A$, and for every $x \in G \backslash\{0\}$ and $y \in M(x), \phi(x)<\theta(y)$. Then whenever $0 \neq z \in[M]_{0}, \theta(z) \in \theta(A)$. 
An ultrafilter $p$ on $S$ is right cancelable if whenever $q, r \in \beta S, q p=r p$ implies $q=r$. An ultrafilter $p \in \mathbb{H}_{\kappa}$ is right cancelable if and only if there is a mapping $M: G \rightarrow p$ such that the subsets $x M(x)$, where $x \in G$, are pairwise disjoint [14, Theorem 4.2].

Proposition 3.11. For every right cancelable ultrafilter $p \in \mathbb{H}_{\kappa}$, the topology $\mathcal{T}[p]$ is zero-dimensional.

Proof. It is immediate from [14, Theorem 4.2] and [13, Theorem 3.2].

A subsemigroup $Q \subseteq S^{*}$ is left saturated (in $\beta S$ ) if for every $x \in \beta S \backslash(Q \cup\{1\}$ ), one has $x Q \cap Q=\emptyset$.

For example, for every $p \in S^{*}, C(p)$ is left saturated. Indeed, if $x q=r$ for some $x \in \beta S$ and $q, r \in C(p)$, then $x q p=r p$, so $x p=p$ and $x \in C^{1}(p)$.

If $\mathcal{T}$ is a regular left invariant topology on $S$, then $\operatorname{Ult}(\mathcal{T})$ is left saturated 14 , Lemma 2.5].

Thus, it follows from Proposition 3.11 that

Corollary 3.12. If $p \in \mathbb{H}_{\kappa}$ is right cancelable, then $\operatorname{Ult}(\mathcal{T}[p])$ is left saturated in $\beta G$.

Note that if $Q$ is a left saturated subsemigroup of $G^{*}$, then for every $p \in Q$, $C(p) \subseteq Q$, and every idempotent right maximal in $Q$ is right maximal in $G^{*}$.

Now we can prove Proposition 3.8 .

Proof of Proposition 3.8. Pick any right cancelable ultrafilter $r \in \mathbb{H}_{\kappa}$ such that $\bar{\phi}(r) \notin \bar{\phi}(C(p))$ and $\bar{\theta}(r)$ is countably incomplete.

This can be done, for example, as follows. Choose a partition $\left\{A_{n}: n<\omega\right\}$ of $\kappa$ such that $\left|A_{n}\right|=\kappa$ for all $n<\omega$ and $\bar{\phi}(C(p)) \subseteq A_{0}$. For each $\alpha<\kappa$, let $x_{\alpha}$ denote the element of $G$ with $\operatorname{supp}\left(x_{\alpha}\right)=\{\alpha\}$. Then any ultrafilter $r$ on $G$ extending the family of subsets

$$
X_{\alpha, n}=\left\{x_{\gamma}: \gamma \geq \alpha \text { and } \gamma \in A_{m} \text { for some } m \geq n\right\},
$$

where $\alpha<\kappa$ and $n<\omega$, is as required.

We claim that $r+p$ is right cancelable and $p \notin(\beta G)+r+p$.

To see that $r+p$ is right cancelable, suppose that $u+r+p=v+r+p$ for some $u, v \in \beta G$. Then by Lemma 3.5 either $u+r \in v+r+C^{1}$ or $v+r \in u+r+C^{1}$, where $C^{1}=C^{1}(p)$. But

$$
\bar{\phi}(u+r)=\bar{\phi}(v+r)=\bar{\phi}(r)
$$

and for every $x \in C(p)$,

$$
\bar{\phi}(u+r+x)=\bar{\phi}(v+r+x)=\bar{\phi}(x) \in \bar{\phi}(C(p)) .
$$

It follows that $u+r=v+r$ and, since $r$ is right cancelable, $u=v$.

To see that $p \notin(\beta G)+r+p$, assume on the contrary that $p=u+r+p$ for some $u \in \beta G$. Then $u+r \in C(p)$, which is a contradiction, since $\bar{\phi}(u+r)=\bar{\phi}(r)$ and $\bar{\phi}(r) \notin \bar{\phi}(C(p))$.

Now let $Q=\operatorname{Ult}(\mathcal{T}[r+p])$. Pick any right maximal idempotent $q \in Q$. By Corollary 3.12, $Q$ is left saturated. Consequently, $C(q) \subset Q$ and $q$ is right maximal in $G^{*}$. By Lemma 3.10, $\bar{\theta}(q)=\bar{\theta}(r+p)=\bar{\theta}(r)$, so $\bar{\theta}(q)$ is countably incomplete. Then by Theorem 3.3, $C(q)$ is finite. By Lemma 3.9, $Q \subseteq(\beta G)+r+p$. For every $x \in(\beta G)+r+p$, one has $x+p=p$. Indeed, $x=u+r+p$ for some $u \in \beta G$ and 
then $x+p=u+r+p+p=u+r+p=x$. It follows that for every $x \in C(q)$, $x+p=x$, so $x \leq_{L} p$. But $p+x \neq p$, since $p+x \in(\beta G)+r+p$ and $p \notin(\beta G)+r+p$. Hence $x<L p$.

Lemma 3.13. Let $p \in \mathbb{H}_{\kappa}$ be an idempotent with finite $C(p)$, and let $q \in \mathbb{H}_{\kappa}$ be a right maximal idempotent such that for each $x \in C(q), x<_{L} p$. Then $p+q$ is an idempotent and $p+q<p$. Furthermore, if $C(q)$ is finite, then $C(p+q) \backslash C(p)$ is a finite right zero semigroup.

Proof. It follows from $q \leq_{L} p$ that $p+q+p+q=p+q+q=p+q$, so $p+q$ is an idempotent. Also $p+q+p=p+q$ and $p+p+q=p+q$, so $p+q \leq p$. Since $q<_{L} p, p+q \neq p$, and consequently, $p+q<p$.

Now let $C(q)$ be finite. Then, by Lemma 3.7. $C(p q)$ is finite as well. By Theorem 3.4 $C(p+q)$ is a chain of right zero semigroups. Let $r \in C(p+q) \backslash C(p)$ and let $K=K(C(p+q))$. Clearly $p+q \in K$. It suffices to show that $r \in K$.

We have that $r+p+q=p+q$. Then by Lemma 3.5, either $r+p \in p+C^{1}(q)$ or $p \in r+p+C^{1}(q)$. The second possibility cannot hold, since it implies that $r+p=p$ and then $r \in C(p)$, which is a contradiction. Consequently, the first possibility holds. Since $r+p=p$ gives a contradiction, it follows that $r+p \in$ $p+C(q)$. For every $x \in C(q)$, we have that $p+x+p+q=p+x+q=p+q$ and $p+q+p+x=p+q+x=p+x$, so $p+C(q) \subseteq K$. Hence $r+p \in K$ and then $r \in K$. The latter follows from the fact that $C(p+q)$ is a chain, and so $C(p+q) \backslash K$ is a subsemigroup.

We now come to the main result of this section.

Theorem 3.14. For every right maximal idempotent $q \in \mathbb{H}_{\kappa}$ with finite $C(q)$ and for every $n \in \mathbb{N}$, there is an idempotent $p \in \mathbb{H}_{\kappa}$ such that $q \in C(p)$ and $C(p)$ is a chain of $n$ finite right zero semigroups.

Proof. If $n=1$, put $p=q$. By Theorem $3.3, C(p)$ is a finite right zero semigroup.

Now let $n>1$ and suppose that we have found an idempotent $p^{\prime} \in \mathbb{H}_{\kappa}$ such that $q \in C\left(p^{\prime}\right)$ and $C\left(p^{\prime}\right)$ is a chain of $n-1$ finite right zero semigroups, say $C_{1}>\ldots>C_{n-1}$. By Proposition 3.8, there is a right maximal idempotent $q^{\prime} \in \mathbb{H}$ with finite $C\left(q^{\prime}\right)$ such that for each $x \in C\left(q^{\prime}\right), x<_{L} p^{\prime}$. Put $p=p^{\prime}+q^{\prime}$. By Lemma 3.13. $p$ is an idempotent, $p<p^{\prime}$ and $C_{n}=C(p) \backslash C\left(p^{\prime}\right)$ is a finite right zero semigroup. It follows that $C(p)$ is the chain $C_{1}>\ldots>C_{n}$.

\section{Proof of Theorem 1.2}

Having proved Theorem 3.14, we can show that

Theorem 4.1. For every $n \in \mathbb{N}$, there is a locally zero-dimensional translation invariant topology $\mathcal{T}$ on $G$ such that $\mathcal{G} \subseteq \mathcal{T}$ and $\operatorname{Ult}(\mathcal{T})$ is a chain of $n$ idempotents.

To prove Theorem 4.1, we need two more lemmas.

Lemma 4.2 ([14, Lemma 2.8]). Let $S$ be a group, let $Q$ be a finite left saturated subsemigroup of $S^{*}$, let $P$ be a subsemigroup of $Q$, and let $\mathcal{T}$ be a left invariant topology on $S$ with $\operatorname{Ult}(\mathcal{T})=P$. Then $\mathcal{T}$ is locally regular if and only if for every $q \in Q \backslash P, q P \cap P \neq \emptyset$ implies $P q \cap P=\emptyset$.

Recall that a space is extremally disconnected if the closure of an open set is open. 
Lemma 4.3 ([14, Lemma 2.3]). Let $\mathcal{T}$ be a left invariant topology on $S$. If $\operatorname{Ult}(\mathcal{T})$ has only one minimal right ideal, then $\mathcal{T}$ is extremally disconnected.

Now we are in a position to prove Theorem 4.1 .

Proof of Theorem 4.1. By Theorem 3.14, there is an idempotent $p \in \mathbb{H}_{\kappa}$ such that $C(p)$ is a chain of $n$ finite right zero semigroups, say $C_{1}>\ldots>C_{n}$. Inductively for each $i=1, \ldots, n$, pick $q_{i} \in C_{i}$ and define $p_{i} \in C_{i}$ by $p_{1}=q_{1}$ and, for $i>1$,

$$
p_{i}=p_{i-1}+q_{i}+p_{i-1} \text {. }
$$

Then $p_{1}>\ldots>p_{n}$. Let $C=C(p)$ and $P=\left\{p_{1}, \ldots, p_{n}\right\}$. We claim that the subsemigroup $P \subseteq C$ possesses the following property:

For every $q \in C \backslash P,(P+q) \cap P=\emptyset$.

Indeed, let $q \in C_{i}$. Then $q \mathcal{R} p_{i}$ and $q \neq p_{i}$. It follows that for every $r \in C$, one has $(r+q) \mathcal{R}\left(r+p_{i}\right)$ and, by Theorem 3.4(1) and Proposition 2.9(i), $r+q \neq r+p_{i}$. If $r \in P$, then $r+p_{i} \in P$, so $r+q \notin P$, since no different elements of $P$ are $\mathcal{R}$-related. Hence $(P+q) \cap P=\emptyset$.

Now let $\mathcal{T}$ be the translation invariant topology on $G$ such that $\operatorname{Ult}(\mathcal{T})=P$. It follows from the property above and Lemma 4.2 that $\mathcal{T}$ is locally regular. Being a chain of idempotents, $P$ has only one minimal right ideal. Hence by Lemma 4.3 , $\mathcal{T}$ is extremally disconnected. Let $X$ be a regular open neighborhood of $0 \in G$ in $\mathcal{T}$. Since extremal disconnectedness is preserved by open subsets and a regular extremally disconnected space is zero-dimensional, we obtain that $X$ is zerodimensional.

Now, using Theorem 4.1 and the Local Monomorphism Theorem [14, Theorem 6.4], we prove Theorem [1.2.

Proof of Theorem 1.2. Let $S$ be an infinite cancellative semigroup with identity, let $|S|=\kappa$, and let $n \in \mathbb{N}$. By Theorem 4.1 there is a locally zero-dimensional translation invariant topology $\mathcal{T}$ on $G$ such that $\mathcal{G} \subseteq \mathcal{T}$ and $\operatorname{Ult}(\mathcal{T})$ is a chain of $n$ idempotents. Pick an open zero-dimensional neighborhood $X$ of $0 \in G$. Every local monomorphism $f: X \rightarrow S$ induces a left invariant topology $\mathcal{T}^{f}$ on $S$ with a neighborhood base at $1 \in S$ consisting of subsets $f(U)$, where $U$ runs over neighborhoods of $0 \in X$ [14, Lemma 2.13]. Clearly, $\operatorname{Ult}(\mathcal{T})$ is a chain of $n$ idempotents in $U(S)$. By the Local Monomorphism Theorem, there is a local monomorphism $f: X \rightarrow S$ such that the topology $\mathcal{T}^{f}$ is zero-dimensional.

Remark 4.4. If $S=G$, the topology $\mathcal{T}$ in Theorem 1.2 can be chosen to be stronger than $\mathcal{G}$, and if $S=\mathbb{R}$, stronger than the natural topology of the real line or even the Sorgenfrey topology (see the proof of [14, Corollary 1.5]).

Topological properties of an almost maximal left topological group $(S, \mathcal{T})$ strongly depend on its ultrafilter semigroup (see [11, Proposition 2.15]). In particular, if $\operatorname{Ult}(\mathcal{T})$ is a chain of $n$ idempotents, then $(S, \mathcal{T})$ is extremally disconnected, irresolvable, and contains nonclosed nowhere dense subsets if $n>1$. Under MA, for each projective $P$ in $\mathfrak{F}$, there is a group topology $\mathcal{T}$ on the countably infinite Boolean group with $\operatorname{Ult}(\mathcal{T})$ isomorphic to $P$ [11, Theorem 5.2]. We conclude the paper with the following question.

Question. Is it true that for each projective $P$ in $\mathfrak{F}$, there exists in ZFC a zerodimensional left invariant topology $\mathcal{T}$ on a group with $\operatorname{Ult}(\mathcal{T})$ isomorphic to $P$ ? 


\section{ACKNOWLEDGEMENT}

The author would like to thank the referee for a careful reading of the paper and for useful comments.

\section{REFERENCES}

1. A. Clifford and G. Preston, The algebraic theory of semigroups, Vol. 1, Mathematical Surveys, no. 7, AMS, Providence, RI, 1961.

2. W. Comfort and S. Negrepontis, The theory of ultrafilters, Springer-Verlag, Berlin, 1974. MR 0396267 (53:135)

3. N. Hindman and D. Strauss, Algebra in the Stone-Čech compactification, De Gruyter, Berlin, 1998. MR 1642231 (99j:54001)

4. N. Hindman, D. Strauss and Y. Zelenyuk, Large rectangular semigroups in Stone-Čech compactifications, Trans. Amer. Math. Soc. 355 (2003), 2795-2812. MR.1975400 (2004g:22001)

5. D. McLean, Idempotent semigroups, Amer. Math. Monthly 61 (1954), 110-113. MR0060505 (15:681a)

6. T. Papazyan, Extremal topologies on a semigroup, Topology Appl. 39 (1991), 229-243. MR 1110567 (92f:22003)

7. I. Protasov, Maximal topologies on groups, Sib. Math. J. 39 (1998), 1184-1194 (Russian). MR:1672661 (99m:22001)

8. W. Ruppert, Compact semitopological semigroups: An intrinsic theory, Lecture Notes in Math. 1079, Springer-Verlag, Berlin, 1984. MR762985 (86e:22001)

9. Y. Zelenyuk, On subsemigroups of $\beta \mathbb{N}$ and absolute coretracts, Semigroup Forum 63 (2001), 457-465. MR1851825 (2002f:22005)

10. Y. Zelenyuk, Weak projectives of finite semigroups, J. Algebra 266 (2003), 77-86. MR.1994529 (2004f:20101)

11. Y. Zelenyuk, Almost maximal spaces, Topology Appl. 154 (2007), 339-357. MR 2278682 (2007i:54072)

12. Y. Zelenyuk, Finite groups in Stone-Čech compactifications, Bull. London Math. Soc. 40 (2008), 337-346. MR2414792 (2009b:22002)

13. Y. Zelenyuk, Topologies on groups determined by discrete subsets, Topology Appl. 155 (2008), 1332-1339. MR2423971 (2009i:54062)

14. Y. Zelenyuk, Regular idempotents in $\beta S$, Trans. Amer. Math. Soc. 362 (2010), 3183-3201. MR 2592952

School of Mathematics, University of the Witwatersrand, Private Bag 3, Wits 2050, SOUTH AFrica

E-mail address: Yevhen.Zelenyuk@wits.ac.za 